\title{
Silent Myocardial Ischemia (SMI) and its Association with Microalbuminuria in Type 2 Diabetes Mellitus (DM)
}

\author{
Shahana Zabeen ${ }^{1}$,Md. Mozammel Hoque ${ }^{2}$, Md. Rezwanur Rahman ${ }^{3}$ \\ ${ }^{1}$ Assistant Professor, Department of Biochemistry, Anwer Khan Modern Medical College, Dhaka, Bangladesh, ${ }^{2}$ Professor, Department of \\ Biochemistry, Bangabandhu Sheikh Mujib Medical University, Dhaka, Bangladesh, ${ }^{3}$ Associate Professor, Department of Biochemistry, \\ Delta Medical College, Dhaka, Bangladesh.
}

\begin{abstract}
:
Background: As silent myocardial ischemia (SMI) is more common in diabetic population leading to the development of future coronary artery disease (CAD), so its early diagnosis is important. SMI can be diagnosed by conventional cardiac stress testing. Presence of SMI can also be suspected by microalbuminuria (MAU) because recently it is claimed that MAU is one of the important predictor for cardiovascular disease. Objective: The study was designed to explore the association between SMI \& MAU in type $2 \mathrm{DM}$. Methods: It was a cross sectional study carried out in the Department of Biochemistry, BSMMU during the period of July 2006 to June 2008. One hundred diagnosed type 2 DM patients were selected from out patient department of BIRDEM. Enrolled study subjects were advised to do ETT and then categorized as ETT + ve \& ETT-ve on the basis of ETT findings. Urinary micro albumin was measured in all study subjects. Unpaired $\mathrm{t}$ test, , chi square test, odds ratio were used to see the level of significance Results: Among the 100 type 2 diabetic subjects of both sexes 50 (male -24, female-26) were ETT + ve designated as type 2 DM with silent MI and rest 50 ( male25 , female-25) were ETT -ve designated as type 2 DM without silent MI. 21 patients (42\%) out of 50 type 2 DM with silent MI \& 16 patients (32\%) out of 50 type 2 DM without silent MI found to have microalbuminuria. Calculated odds ratio was 1.5. Conclusion: Microalbuminuria is a possible risk factor for SMI in type $2 \mathrm{DM}$. Urinary micro albumin can be used particularly as a screening test for early detection of SMI.
\end{abstract}

Key word: SMI (Silent myocardial ischemia), MAU (Microalbuminuria), Type 2 DM.

[BSMMU J 2012; 5(1):42-45]

\section{Introduction:}

Diabetes is a common and serious disease leading to chronic, mostly irreversible macro and microvascular complications. Microvascular complications include retinopathy, neuropathy, and nephropathy. Macrovascular complications include cardiovascular, cerebrovascular, and peripheral vascular disease. ${ }^{1}$ Coronary artery disease (CAD) is one of the macrovascular complication accounting for as many as $80 \%$ of death in diabetic patients, furthermore the prevalence of silent myocardial ischemia among individuals with DM is high ranging from $20 \%$ to $>50 \%{ }^{2}$

Myocardial ischemia is defined as a transient and reversible decrease in oxygen to myocardium, leading to an inadequacy of contribution/ need ratio. It is responsible for the following sequential events: initially haemodynamic changes followed by kinetic changes (elevated end diastolic left ventricular pressure), metabolic (lactate production), electrical (repolarisation change) and clinical

Address for Correspondence: Dr. Shahana Zabeen, Assistant Professor, Department of Biochemistry, Anwer Khan Modern Medical College, Dhaka, Bangladesh. (chest pain) events. Silent myocardial ischemia (SMI) is characterized by lack of final clinical event. ${ }^{3}$

Silent myocardial ischemia is particularly concerned in diabetic patients. It is suspected that partial or complete autonomic denervation in diabetic causes impaired symptom perception e.g. lack of recognition of chest pain leading to SMI. ${ }^{4}$

Silent myocardial ischemia (SMI) can be detected by using cardiac stress testing. SMI has been defined as exercise induced ST depression of $>1 \mathrm{~mm}$ in the absence of CAD symptoms $^{5}$. Patients with a resting ECG suggestive of ischemia or infarction should undergo stress testing independent of previous or ongoing symptoms. In addition, asymptomatic patients with diabetes and two or more cardiovascular risk factors should also undergo stress testing. These risk factors include: total cholesterol $>240 \mathrm{mg} / \mathrm{dl}, \mathrm{LDL}>160 \mathrm{mg} / \mathrm{dl}, \mathrm{HDL}<35 \mathrm{mg} / \mathrm{dl}, \mathrm{BP}>140 / 90 \mathrm{~mm}$ $\mathrm{Hg}$, smoking, family history of premature CAD and microalbuminuria or proteinuria. ${ }^{4}$

Microalbminuria in type 2 DM reflects an underlying predisposition to developing progressive kidney diseases as well as serving as a marker of predilection for generalized 
cardiovascular disease. ${ }^{6}$ Persistent microalbuminuria has also been associated with an increase risk of atherosclerosis and cardiovascular mortality. Therefore, patients with diabetes should be screened regularly for microalbuminuria. ${ }^{7}$ Silent myocardial ischemia leading to development of future CAD is common in Type $2 \mathrm{DM}$ but more common in Type $2 \mathrm{DM}$ with microalbuminuria. Therefore, microalbuminuria can be used as an early marker as well as for risk stratification of cardiovascular disease. ${ }^{8}$

Microalbuminuria is defined as low level of urinary albumin excretion of 30 to $300 \mathrm{mg} /$ day or $20-200 \mu \mathrm{g} / \mathrm{min}$ in which it gives the urinary concentration of albumin $20-200 \mathrm{mg} / \mathrm{dl}$. 9,11 The possible pathophysiologic link between microalbuminuria and atherosclerosis is the increased generalized transvascular leakage of albumin. It is hypothesized that the systemic transvascular leakiness may also include lipoproteins, thus allowing for increased lipid penetration into the vessel wall. The leakiness might be due to hemodynamic factors or structural or functional perturbations of the endothelium or the intracellular matrix beneath. ${ }^{10}$

Coronary artery disease (CAD) is the most common form of heart disease and the single most important cause of premature death in all regions of the world. Since the silent myocardial ischemia (SMI) is more common in diabetic population therefore identification of SMI in many asymptomatic type 2 diabetic patients are important for the prevention of future myocardial infarction.

SMI is diagnosed by conventional cardiac stress testing. Presence of SMI can also be suspected by microalbuminuria (MAU) because recently it is claimed that as an early marker microalbumin, may be a useful indicator for early detection of SMI particularly for screening purpose.

\section{Methods:}

It was a cross sectional study carried out in the Department of Biochemistry, BSMMU during the period of July 2006 to June 2008. Diagnosed type 2 DM patients were selected from out patient department of BIRDEM.

Enrolled study subjects were advised to do ETT and fifty subjects were selected randomly as ETT +ve (with silent myocardial ischemia) \& fifty as ETT -ve (without silent myocardial ischemia) on the basis of ETT findings. Exercise ECG (ECG during ETT) was performed by using 12 standard leads on a treadmill according to Bruce protocol. The protocol had seven stages, each lasting 3 min resulting in 21 minute exercise for a complete test ${ }^{12}$.

The exercise test was considered positive if there was a horizontal and down slopping ST segment depression of at least $1 \mathrm{~mm}$ occurring at least $0.08 \mathrm{~s}$ after the $\mathrm{J}$ point. The test was considered negative when the heart reached the maximal predicted exercise heart rate (calculated without a change in ST segment with the ASTRAND formula, 220age) without a change in ST segment ${ }^{12}$.

For micro albumin desired amount of urine was collected from ETT +ve \& ETT-ve patients. Urinary micro albumin concentration was measured by immunometric assay ${ }^{9}$. Microalbuminuria is defined as urinary albumin excretion of 30 to $300 \mathrm{mg} /$ day or $20-200 \mu \mathrm{g} / \mathrm{min}$.Chi square test, unpaired t test and odds ratio were used to see the level of significance.

\section{Results:}

Table I shows grouping of the study subjects with age \& sex distribution. Unpaired t test \& Chi square test done but no significant differences found between two groups with respect to age \& sex.

Table-I

Age \& sex frequency of study subjects $(n=100)$

\begin{tabular}{lcccc}
\hline Parameter & $\begin{array}{c}\text { Type II DM } \\
\text { with SMI } \\
\mathrm{n}=50\end{array}$ & $\begin{array}{c}\text { Type II DM } \\
\text { without SMI } \\
\mathrm{n}=50\end{array}$ & $\begin{array}{c}\text { P- } \\
\text { value }\end{array}$ \\
\hline Age (Mean \pm SD) & $50.44 \pm 8.26$ & $47.26 \pm 8.39$ & $>0.05$ \\
Sex $\quad$ Male (No.) & 24 & 25 & $>0.05$ \\
& Female(No.) & 26 & 25 & \\
\hline
\end{tabular}

P-value reached by Chi-Square test and unpaired $t-$ test.

The mean $\pm \mathrm{SD}$ of microalbuminuria in type $2 \mathrm{DM}$ with silent MI was $103.31 \pm 66.287$ with the range of $21-198 \mathrm{mg} /$ $\mathrm{dl}$ and in without silent MI $87.90 \pm 74.113$ with the range of 21-190 mg/dl respectively (Table II).

Table II

Microalbuminuria in the study subjects $(n=100)$

\begin{tabular}{lcccc}
\hline Parameter & \multicolumn{2}{c}{ Type 2 DM with silent $\mathrm{MI}(\mathrm{n}=50)$} & \multicolumn{2}{c}{ Type 2 DM without silent MI (n=50) } \\
& Mean \pm SD & Range & Mean \pm SD & Range \\
\hline Microalbuminuria $(\mathrm{mg} / \mathrm{dl})$ & $103.31 \pm 66.287$ & $21-198$ & $87.90 \pm 74.113$ & $21-190$ \\
\hline
\end{tabular}


In table III microalbuminuria was evaluated as a risk factor to develop SMI in type 2 DM . Twenty one (42\%) patients out of 50 type 2 DM with silent MI \& sixteen (32\%) patients out of 50 type $2 \mathrm{DM}$ without silent $\mathrm{MI}$ found to show microalbuminuria. Calculated odds ratio was 1.5.

\section{Table-III}

Association of Microalbuminuria with silent MI $(n=100)$

\begin{tabular}{lccc}
\hline $\begin{array}{l}\text { Microalbuminuria } \\
\text { mg/l(20-200) }\end{array}$ & $\begin{array}{c}\text { Type 2 DM } \\
\text { with silent MI } \\
(\mathrm{n}=50)\end{array}$ & $\begin{array}{c}\text { Type 2 DM } \\
\text { without silent MI } \\
(\mathrm{n}=50)\end{array}$ & $\begin{array}{c}\text { Odds } \\
\text { ratio }\end{array}$ \\
\hline Present & $21(42 \%)$ & $16(32 \%)$ & 1.5 \\
Absent & $29(58 \%)$ & $34(68 \%)$ & \\
\hline
\end{tabular}

\section{Discussion:}

Cardiovascular disease is the leading cause of death among people with diabetes mellitus. Silent myocardial ischemia (SMI) is particularly concerned in diabetic patients. Recently many studies have identified microalbuminuria (MAU) as a possible risk factor for cardiovascular morbidity and mortality in people with diabetes besides other conventional risk factors such as hypertension ,dyslipidaemia and smoking. 5,13 Diabetic patients with MAU have 8-15 times higher cardiac mortality than diabetic patients without MAU. A recent meta-analysis revealed that, in people with diabetes, MAU is associated with an odds ratio of an 2.1 for cardiovascular mortality ${ }^{14}$.

Patients with SMI have more frequent adverse cardiac events than patients without SMI.

In this cross sectional study our attempt was to demonstrate an association between SMI \& MAU. Our study has revealed MAU as a possible risk factor for SMI in type $2 \mathrm{DM}$ with OR of 1.5. Almost similar findings were observed in the study of Corona et al. $2005^{15}$ (OR 1.90), Muthu et al. $2008^{16}$ (OR 1.38), Yuyun et al. $2004^{10}$ (OR 1.40), Lowry, $2007^{17}$ (OR 1.96), \& Weir, $2007^{18}$ (OR 1.43).

In the study of Corona et al (2005) 215 (13.6\%) out of 1586 enrolled subjects had microalbuminuria and 1371 (86.4\%) did not. During follow-up, 10patients with microalbuminuria \& 31 patients without microalbuminuria developed an acute MI. In a 4 yr follow up study on 804 type 2 diabetic patients Muthu et al (2008) found both type 2 diabetes and MAU to increase the risk of new cardiovascular events in univariate analysis. From multivariable models, presence of diabetes remained significantly and independently related to incident CHD. Presence of MAU also remained significantly \& independently related to incident CHD with the OR $1.38 .{ }^{16}$
This appears to be permissive to our findings that MAU is associated with SMI .In EPIC - NORFOLK study (19932002) researchers investigated the prognostic significance of albuminuria with baseline CHD events. They found MAU to be associated with total mortality with OR 1.61. Another study demonstrated a significant association between MAU \& SMI in a cohort of 1576 Native Americans with diabetes. Later the LIFE study confirmed that the $\mathrm{CAD}$ risk associated with other conventional risk factors becomes more than doubled in the presence of MAU ${ }^{18}$. However it is also becoming increasingly recognized as an independent risk factor for cardiovascular disease in patients with or without diabetes. The recent stenostudy has demonstrated that multifactorial interventions can significantly reduce cardiovascular events in microalbuminuric patients with type 2 diabetes. ${ }^{19}$

\section{Conclusions:}

SMI can be suspected by the presence of MAU in type 2 DM. Therefore MAU can be used particularly as a screening test for early detection of SMI. Early detection of SMI in type $2 \mathrm{DM}$ can prevent future CAD which is the most frequent cause of death in type 2 diabetic patients.

\section{References:}

1. Donelly R, Emsile-Smith AM, Gardner ID \& Morris AD. ABC of arterial and venous disease-vascular complication of diabetes. BMJ 2002; 320: 1062-1066.

2. Ali YS \& Maron DJ. Screening for coronary disease in diabetes: When \& How? Clinical diabetes 2006; 24:169-173.

3. Barthelemy O, Feuvre CL, Timsit J. Silent myocardial ischemia screening in patients with diabetes mellitus. Arquivos Brasileiros de Endocrinologia \& Metbologia 2007; 51: 304308.

4. Tabibiazar R \& Edelman SV. Silent ischemia in people with diabetes: A condition that must be heard. Clinical Diabetes 2003; 21: 5-9.

5. Rutter MK, Wahid ST, McComb JM \& Marshall SM. Significance of silent ischemia and microalbuminuria in predicting coronary events in asymptomatic patients with type 2 diabetes. J Am Coll Cardiol 2002; 40: 56-61.

6. Hussain AR, Khan MA, Ali AK \& Vaales S. Type 2 diabetes in rural and urban population: diverse prevalence and associated risk factors in Bangladesh. Diabetes Med 2005; 22: 931-936.

7. Goddard J, Turner AN, Cumming AD, Stewart LH. Kidney and urinary tract disease. NA Boon, NR colledge, BR walker \& JAA Hunter (eds), Davidson's Principles \& Practice of Medicine, $20^{\text {th }}$ edn, Churchill livingstone Elsevier, Philadelphia pp. 805-848.

8. Stehouwer CDA \& Smulders YM .Microalbuminuria and risk for cardiovascular disease: Analysis of potential mechanisms. J Am Soc Nephrol 2006; 17: 2106-2111. 
9. Zeeuw Dde, Parving HH \& Henning RH. Microalbuminuria as an early marker for cardiovascular disease. J Am Soc Nephrol 2006; 17: 2100-2105.

10. Yuyun MF, Khaw KTee, Luben R, Wlech A, Bingham Sheila Day NE \& Wareham, NJ. A Prospective study of Microalbuminuria and Incident Coronary Heart Disease and its prognostic significance in British population. Am J Epidemiol 2004; 159: 284-293.

11. Crook MA. Carbohydrate Metabolism', Clinical Chemistry and Metabolic Medicine, $7^{\text {th }}$ (edn), Martin A Crook, Lewisham, London, p 186.

12. Delenne BJ, Savin B, Habib G, Bory M, Vague P \& Lassmann V. Silent Myocardial Ischemia in Patients with Diabetes. Diabetes care 1999; 22: 1396-1400.

13. Davis T, Fortun P, Mulder J, Davis W\& Bruce D. Silent myocardial ischemia and it's prognosis in a community based cohort of type 2 diabetic patients. Diabetologia 2004; 47: 395-399.
14. Cooper S \& Caldwell JH .Coronary Artery Disease in people with Diabetes: Diagnostic and risk factor evaluation. Clinical Diabetes 1999; 17:no.2, Retrived 1999, from http://Journal. Diabetes. Org / clinical diabetes.

15. Corona AJ, Martinez DR, Avila MH, Simon J. Microalbuminuria as a predictor of myocardial ischemia in a Mexican population. NCBL; 2005:97: 34-39.

16. Muthu S, Visseren F, Algra A. The impact of type 2 DM and microalbuminuria on future cardiovascular events (SMART) study. 2008; 25: 51-57.

17. Lowry F. Microalbuminuria: An increasingly recognized risk factor for CVD. Retrived 2007, from http:// WWW. The heart. Org/ article.

18. Weir MR. Microalbuminuria and cardiovascular disease. Cli J Am Soc Nephrol 2007; 2: 581-590.

19. MacIsacc RJ, Cooper ME. MAU and diabetic cardiovascular disease. Current atherosclerosis reports 2007; 5: 350-357. 